\title{
Keine Verbesserung von Gesamtüberleben und Lebensqualität versus Standardtherapie
}

Fragestellung: Finden sich in der Subgruppe der Patienten mit Glioblastom ohne MGMT-Promotormethylierung Hinweise auf eine Überlegenheit von Bevacizumab plus Irinotecan gegenüber Temozolomid in der Primärtherapie?

Hintergrund: Standardtherapie des Glioblastoms nach der Operation ist die Strahlentherapie mit begleitender und nachfolgender Chemotherapie mit Temozolomid. Sicher profitieren von dieser zusätzlichen Chemotherapie aber nur die Patienten, deren Tumoren eine Methylierung der Promotorregion des O6Methylguanin-DNA-Methyltransferase (MGMT-Gens) aufweisen, weil diese vermutlich weniger MGMT-Protein im Tumor exprimieren und deshalb DNA-Schäden schlechter reparieren können. Aus diesem Grund bietet sich die große Gruppe der Patienten ohne MGMT-Promotormethylierung an, im Rahmen einer klinischen Studie ein experimentelles Regime ohne Temozolomid zu prüfen und mit dem in dieser Patientenpopulation kaum wirksamen Temozolomid zu vergleichen. Diesen Weg ging auch die GLARIUS-Studie, die in der Primärtherapie Bevacizumab, den Antikörper gegen den vaskulären endothelialen Wachstumsfaktor (VEGF),

Herrlinger U, Schäfer N, Steinbach JP et al. Bevacizumab plus irinotecan versus temozolomide in newly diagnosed 06-methylguanine-DNA-methyltransferase non methylated glioblastoma: The randomized GLARIUS Trial. J Clin Oncol 2016; 34: $1611-9$ mit dem beim Glioblastom als Monotherapie nicht wirksamen Topoisomerasehemmstoff Irinotecan kombinierte. Ziel war es, Hinweise $\mathrm{zu}$ erhalten, dass dieses Bevacizumab-haltige Regime in der Subgruppe der Patienten mit Tumoren ohne
MGMT-Promotermethylierung dem Temozolomid überlegen sein könnte.

Patienten und Methodik: Es handelte sich um eine nicht verblindete Phase-II-Studie, in der 182 Patienten in 22 Zentren 2:1 zur Kombination von Bevacizumab $10 \mathrm{mg} / \mathrm{kg}$ alle zwei Wochen während der Strahlentherapie, gefolgt von einer Erhaltungstherapie mit Bevacizumab plus Irinotecan $\left(125 \mathrm{mg} / \mathrm{m}^{2}\right.$ alle zwei Wochen) oder aber der Temozolomid-Standardtherapie mit Strahlentherapie randomisiert wurden.

Der primäre Endpunkt war das progressionsfreie Überleben nach sechs Monaten.

Ergebnisse: In der modifizierten Intention-to-treat-Population war das progressionsfreie Überleben mit 79,3\% nach sechs Monaten im experimentellen Arm deutlich höher als mit 42,6\% im Standardarm. Das mediane progressionsfreie Überleben war mit 9,7 gegenüber 5,99 Monaten im experimentellen Arm verbessert. Zum Zeitpunkt der Progression erhielten 81,8\% aller Patienten, die mit einer zweiten Therapie behandelt wurden, Bevacizumab. Das Gesamtüberleben unterschied sich in den beiden Armen nicht: Es betrug 16,6 Monate im experimentellen Arm und 17,5 Monate im Kontrollarm. Auch bei der Lebensqualität ergaben sich keine relevanten Unterschiede zwischen den Armen.

Schlussfolgerung: Die Autoren vermuten, dass am ehesten die hohe Crossover-Rate, die im Studiendesign eingeplant war, dazu führte, dass sich der Zugewinn an progressionsfreiem Überleben nicht auch beim Gesamtüberleben zeigte.

\section{Von der Angiogenesehemmung ist kein Durchbruch mehr zu erwarten}

Nach den negativen Phase-III-Studien AVAglio und RTOG 0825 zeigt eine weitere Studie zu Bevacizumab in der Primärtherapie des Glioblastoms das gleiche Muster: einen Zugewinn an progressionsfreier Überlebenszeit, nicht aber einen Effekt auf das Gesamtüberleben. Dieses Ergebnis kann angesichts der Ergebnisse der großen Phase-III-Studie nicht überraschen, es sei denn, man hätte postuliert, dass Irinotecan eine relevante zusätzliche Wirkung entfaltet. Im Studiendesign der GLARIUSStudie hatte die Hinzunahme von Irinotecan am ehesten historische Gründe und war ebenso wenig begründet wie der primäre Einsatz der Kombination der beiden Substanzen in den frühen Studien im Rezidiv vor vielen Jahren. Die Stärke der GLARIUS-Studie lag in dem Ansatz, primär eine Patientenpopulation herauszuwählen, die eher nicht von Temozolomid profitiert, so dass a priori die Chancen nicht schlecht waren, hier eine Überlegenheit zu zeigen. Dass dies nicht gelang, mag tatsächlich zum Teil am Crossover liegen, zum Teil aber vielleicht auch nur einfach daran, dass sich die Überlebenszeit beim Glioblastom durch Hemmung der VEGF-abhängigen Angiogenese nicht verbessern lässt. Damit wird der klinische Wert für die Lebensqualität, den Bevacizumab bei vielen Patienten vor allen Dingen in der Spätphase der Erkrankung hat, nicht infrage gestellt.

Zusammengefasst liegt eine bis auf den Einsatz von Irinotecan gut geplante und kompetent durchgeführte Studie vor, deren Ergebnisse wie alle anderen Studien zur Angiogenesehemmung beim Glioblastom der letzten Jahre enttäuschen, und veranschaulichen, dass von Angiogenesehemmung allein beim Glioblastom möglicherweise kein großer Durchbruch mehr zu erwarten ist. 